\title{
In vitro, in vivo and in silico drug- drug interaction study between Vildagliptin and Bisoprolol fumarate
}

\author{
Kazi Ashfak Ahmed Chowdhury, Mohammad Shah \\ Hafez Kabir, Mohammed Munawar Hossain, Tanvir \\ Ahmad Chowdhury and Nishan Chakrabarty \\ Department of Pharmacy \\ International Islamic University Chittagong, Bangladesh
}

\begin{abstract}
Aim of the present study is to evaluate the in vitro, in vivo and in silico drug-drug interaction between Vildagliptin and Bisoprolol fumarate. Interaction between Vildagliptin and Bisoprolol fumarate has been studied in a system of water at a fixed $37^{\circ} \mathrm{C}$ at $\mathrm{pH} 1.0,3.0$ and 6.8 by using spectral observation, Job's method of continuous variation, Ardon's method. In silico complexation was measured by NMR prediction and DDI-CPI analysis. From spectrophotometric study, Vildagliptin and Bisoprolol fumarate give different spectra when Vildagliptin mixed with Bisoprolol fumarate in 1:1 mixture, intensity of spectra of Vildagliptin transform surprisingly due to interaction. The jobs plot was attained by plotting

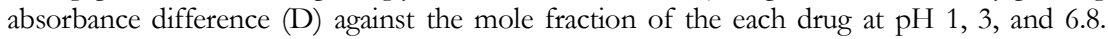
Vildagliptin moderate for 1:1 mixture with Bisoprolol fumarate and slightly lower spectra indicate the formation of 1:1 mixture of Vildagliptin with Bisoprolol fumarate. The value of stability constant for the drug-drug system at $\mathrm{pH} 1.0,3.0$ and 6.8 are 1.8750 .7778 and 1.2000 respectively. From the IR report it also proved that Vildagliptin and Bisoprolol fumarate produced interaction. In this OGTT test at 1:1, 1:2 and 2:1 complex blood glucose level decrease compared to Vildagliptin. Because when drug-drug interaction happened, then drug cannot exhibit its main activity. That's why in this test complex showed different activity. In in silico methods, results showed that the drugs interact with each other. So, a careful concern is desired during simultaneous administration of Vildagliptin with Bisoprolol fumarate.
\end{abstract}

Keywords Drug-drug interaction, Vildagliptin, Bisoprolol fumarate, Ardon's, Jobs method, OGTT.

Paper type Research paper

\section{Introduction}

It is often necessary to take more than one drugs at a time and some level of medication collaboration happens with attending utilization of medications. Drug interactions can cause serious harm to the patient, sometimes causes serious adverse reactions and nowadays become an obvious concern for the health

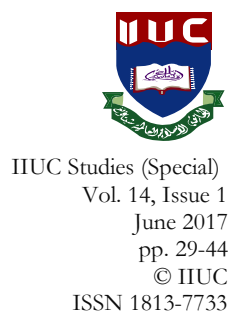




\section{IIUC STUDIES, 14 (Issue 1, Special)}

care providers. For instance, drug interactions, especially with medications having a narrow therapeutic range, may have serious adverse outcomes. A literature search in Medline and Embase database from 1990 to 2006 illustrated that drug-drug interactions (DDIs) were detained accountable for $0.054 \%$ of the emergency department (ED) visits, $0.57 \%$ of the hospital admissions, and $0.12 \%$ of the re-hospitalizations (Becker et al., 2007). It is conceivable that medication connection can be helpful or hindering. The utilization of various medications may give synergism, for example, expanding the viability of helpful impact, diminishing measurement however holding the same adequacy to maintain a strategic distance from poisonous quality, or minimizing the medication resistance (Chou, T. C.,2006). Drug-drug interactions are the important cause of therapeutic problems and increased number of hospital admissions within Asia, where Bangladesh has a leading position. It is because of lack of knowledge about it. Both physicians and medicine taker (patient) are not concern it. As a result, these problems rising dangerously. The United States (US) withdraw half of drugs from the market, because of safety reasons, during the period 1999-2003, was associated with important drug interactions (Huang, S. M. \& Lesko, L. J. ,2004). The unfavorable impacts of medications which are an outcome of drug interactions can be averted by making proper move. It was estimated that more than three-quarters of clinically significant drug interactions can be avoided (Van et al.,2005). For adequate management of drug-drug interactions, the access to appropriate information sources is very important for health professionals (Bergk, et al.,2005). But problem seriously arise when new drugs come in the market, where lack of information included in its descriptions. So, drug-drug interaction (DDI) or drug-food interaction (DFI) is challenging sector, where huge amount of medicine are present in the market. Bur multiple drug treatment is a typical and helpful practice for the treatment of ailments where two or more medications are given in the meantime or simultaneously. For this reason DDI should measure selective group of drugs with new medicine.

The prime object of this project was to clarify the potential drug-drug interactions (DDIs) as a contributing factor towards drug safety because these drugs have narrow TI and any kind of potential interaction may leads to toxic or sub-therapeutic effect. The main focus of this work was to identify whether there is any interaction between Vildagliptin (antidiabetic drug) and Bisoprolol fumarate (beta-blockers) present or not. The present study was to find out the DDIs as well as to establish the stability of the complexes, which could be formed after interaction between Vildagliptin and Bisoprolol fumarate at various $\mathrm{pH}$. The values of stability 
In vitro, in vivo and in silico 31

constants were determined by using Job's continuous-variation analysis, Ardon's spectrophotometric measurement methods and IRAffinity-1S FTIR spectrophotometer. DDI also measured with in vivo Oral glucose tolerant test (OGTT). In silico complexation was measured by NMR prediction and DDI-CPI analysis.

\section{Materials and Methods}

\subsection{Drugs and chemicals}

Vildagliptin and Bisoprolol fumarate were collected from a renowned pharmaceutical company. All chemicals and reagents were of analytical grade.

\subsection{Equipment}

UV-visible spectrophotometer, $\mathrm{pH}$ meter (Hanna-HI981.7, USA), analytical balance (Model No. LF 224 DR, Shinko Denshi Co Ltd, Japan), a thermostatic water bath (Shimadzu, Japan), IR Affinity-1S spectrophotometer (Shimadzu, USA) and Rapid View ${ }^{\mathrm{TM}}$ (California, USA) were used for the test.

\subsection{Preparation of standard solutions}

Vildagliptin $\left(1 \times 10^{-3} \mathrm{M}\right)$ and Bisoprolol fumarate $\left(1 \times 10^{-4} \mathrm{M}\right)$ were dissolved in distilled water to prepare the stock solutions.

\subsection{In vitro studies}

\subsubsection{Absorbance Spectra Analysis}

In observation of the spectra, the absorption characteristics of Vildagliptin and Bisoprolol fumarate and their 1:1 mixture in the solutions of buffers (6) $\mathrm{pH} \mathrm{1.0,3.0} \mathrm{and} 6.8$ were contrasted and those of each interacting species. The concentrations of the specimen were kept at extremely dilute levels for every situation and the estimations made utilizing UV-VIS spectrophotometer. The spectra of the working standard arrangements $(1 \times 10-3 \mathrm{M})$ were recorded between $400-200 \mathrm{~nm}$. The spectra were contrasted and those of the immaculate examples for every situation.

\subsubsection{Job's Spectrophotometric Method}

According to this method (Job.,Ann Chim,1928), (Mohiuddin et al. 2009) the absorbance of sequence of Vildagliptin $\left(1 \mathrm{X}^{10^{-3}}\right)$ with Bisoprolol fumarate $\left(1 \times 10^{-4}\right)$ in different molar ratios $1: 9,2: 8,3: 7,4: 6,5: 5,6: 4,7: 3$, $8: 2$, 9:1 were measured by keeping the total mole consistent. The 


\section{IIUC STUDIES, 14 (Issue 1, Special)}

absorbances of solutions were measured at $244 \mathrm{~nm}$ utilizing UV-VIS spectrophotometer. The watched absorbance of the mixtures at different mole fractions was subtracted from whole of the qualities with the expectation of free drugs (Vildagliptin and Bisoprolol fumarate). The absorbance difference (D) was then plotted against the mole fractions of the medication in the mixtures. On the off chance that the development consistent is sensibly great, two straight lines of various inclines that meet at a mole proportion that relates to the consolidating proportion in the complex are gotten.

\subsubsection{Ardon's spectrometric method}

In this method, (Ardon,M.,1957, Sayeed, M. A. \& Rana,S.,2013) concentrations of Vildagliptin was diverse while observance the concentrations of Bisoprolol fumarate fixed at $1 \times 10^{-4} \mathrm{M}$. The study performed in buffer at $\mathrm{pH}$ 1.0, 3.0 and 6.8. The absorbances of solutions were measured at $244 \mathrm{~nm}$ using UV-VIS spectrophotometer. The Ardon's equation was used is given below-

$\begin{array}{ccc}1 & 1 & 1 \\ \left(\mathrm{D}-\in_{\mathrm{A}} \mathrm{C}\right) & \mathrm{KC}\left(\in_{\mathrm{com}}-\in_{\mathrm{A}}\right)(\mathrm{B})^{\mathrm{n}} & \mathrm{C}\left(\in_{\mathrm{com}^{-}} \in \in_{\mathrm{A}}\right)\end{array}$

Where, $\mathrm{D}=$ Absorbance of the mixture, $\mathrm{B}=$ Molar concentration of the Vildagliptin. $\mathrm{C}=$ Molar concentration of the Bisoprolol fumarate $\epsilon_{\text {com }}=$ Molar extinction co-efficient of the complex, $\epsilon_{\mathrm{A}}=$ Molar extinction co-efficient of the Vildagliptin.

The value of $\mathrm{n}$ was chosen as 1 , which is an essential condition for validation of the method. The value for $1 /\left(\mathrm{D}-\epsilon_{\mathrm{A}} \mathrm{C}\right.$ ) was plotted versus 1 / D to get the straight lines.

The stability constant of the complex was given by the relation, $\mathrm{K}=$ intercept / slope

It is to be mentioned that this method is only valid for the systems where 1:1 complexes are found.

\subsubsection{Drug-drug interaction measured by IRAffinity-1S FTIR spectrophotometer}

The IRAffinity-1S FTIR spectrophotometer has been planned in view of educating and general research labs. Notwithstanding its conservative plan, it offers full usefulness for all FTIR strategies, including transmission, diffuse reflection, and attenuated total reflection (ATR). 
In vitro, in vivo and in silico 33

Spectra were recorded on IRAffinity-1S FTIR spectrophotometer (Shimadzu, USA) apparatus in the range of $350-4600 \mathrm{~cm}^{-1}$. The spectrum was a mean of ten consecutive scans on the same sample. Spectra recorded for Vildagliptin, Bisoprolol and their 1:1 complex.

\subsection{In vivo study}

\subsubsection{Hypoglycemic effect in glucose induced hyperglycemic mice (OGT'T)}

This test was performed according to the established method (Xia, F. et al., 2013) with minor modification. $1^{\text {st }}$ group was treated as normal control group, $2^{\text {nd }}$ group treated with Vildagliptin $\left(50 \mathrm{mg} / \mathrm{kg}\right.$, p.o.), $3^{\text {rd }}$ group were treated with Bisoprolol fumarate $\left(50 \mathrm{mg} / \mathrm{kg}\right.$, p.o.), $4^{\text {th }}$ group were treated with Vildagliptin $(50 \mathrm{mg} / \mathrm{kg}$, p.o.) + Bisoprolol fumarate $(50 \mathrm{mg} / \mathrm{kg}$, p.o.) of 1:1 mixture, $5^{\text {th }}$ group were treated with 1:2 mixture, and $6^{\text {th }}$ group were treated with 2:1 mixture respectively. Glucose solution $(1 \mathrm{~g} / \mathrm{kg})$ was administered at first. Then drugs and complex solutions were administered to the glucose fed mice. Serum glucose level of blood test from tail vein was evaluated by utilizing Glucometer (Rapid View ${ }^{\mathrm{TM}}$ ) at 0 , $60 \mathrm{~min}, 120 \mathrm{~min}$, and $180 \mathrm{~min}$. Percent decrease of blood glucose level after 120 min measured by following equation,

$\%$ decrease $=\frac{G L_{0 \min }-G L_{180 \min }}{G L_{0 \min }} \times 100$

$G L_{0 \min }=$ Blood Glucose level at $0 \mathrm{~min}, G L_{180 \min }=$ Blood Glucose level at $180 \mathrm{~min}$.

\subsection{In silico studies}

\subsubsection{Structural studies}

Vildagliptin and Bisoprolol fumarate were acquired from Pubchem compound vault (http://www.ncbi.nlm.nih.gov/pccompound). The structures were drawn utilizing the Chem sketch package 11.0 having a place with the ACD chem. Lab. Then the structures opened with the Accelrys Discovery Studio Visualizer and performed the interaction. The found complex molecule of Vildagliptin and Bisoprolol fumarate then saved. 
34 IIUC STUDIES, 14 (Issue 1, Special)

\subsubsection{NMR Prediction}

H-1 and C-13 NMR prediction was done by Chem office 2002.

\subsubsection{DDI-CPI analysis}

Users need to upload a drug molecule in mol2 organize with charges and hydrogens included. At the point when the client presents a medication atom, our server checks the arrangement appropriateness and ascertains the association profile of this medication towards all existent drugs (Luo, H. et al. ,2011).

\subsection{Statistical analysis}

Statistical programs used were GRAPHPAD PRISM ${ }^{\circledR}$, which also used for graph preparation. The outcomes were factually broke down utilizing rehashed measures analysis of variance with Dunnett's test by using SPSS (version 22.0). $\mathrm{P}<0.05$ and $\mathrm{P}<0.01$ were considered as statistically significant.

\section{Results}

\subsection{Spectral study}

The drugs concentrated on indicated retention in UV-VIS area. The atomic types of Vildagliptin when mixed with Bisoprolol fumarate demonstrated a few changes in assimilation qualities of this molecule (Bisoprolol fumarate) incorporating a few moves in the ingestion maxima. Consequently modification in ghastly example might be viewed as a pointer for the essential communication among these drugs (Figure 1).

\subsection{Study of Job's method}

The molar proportions of the complexes of Vildagliptin with Bisoprolol fumarate were assessed by Job's method for consistent variety. The watched absorbance qualities were measured in $\mathrm{pH} 1.0,3.0$ and 6.8 at different focuses $\left(0.1 \times 10^{-3}\right.$ to $\left.0.9 \times 10^{-3} \mathrm{M}\right)$ Vildagliptin with Bisoprolol fumarate of at $244 \mathrm{~nm}$. The Job's plots at $\mathrm{pH} \mathrm{1.0,} 3.0$ and 6.8 were acquired by plotting absorbance contrasts against the mole part of the medication (Vildagliptin) which are exhibited in Figure 2. 
In vitro, in vivo and in silico 35

\subsection{Effect of Vildagliptin on Bisoprolol fumarate using Ardon's method}

Ardon's plot confirmed the formation of 1:1 complex of Vildagliptin and Bisoprolol fumarate at $\mathrm{pH} 1.0,3.0$ and 6.8 , since the method is valid only for 1:1 complexes. The values of $1 /$ (drug) by using the Ardon's equation:

$\begin{array}{ccc}1 & 1 & 1 \\ \left(\mathrm{D}-\epsilon_{\mathrm{A}} \mathrm{C}\right) & \mathrm{KC}\left(\in_{\mathrm{com}^{-}} \in_{\mathrm{A}}\right)(\mathrm{B})^{\mathrm{n}} & \mathrm{C}\left(\in_{\mathrm{com}^{-}} \in_{\mathrm{A}}\right)\end{array}$

The data for Ardon's gave straight lines with intercept which are presented in Figure 3 indicates the formation of 1:1 complexes for the system at both $\mathrm{pH}$.

\subsection{Estimation of Stability Constant}

The value of stability constant for the complexation of Vildagliptin with Bisoprolol fumarate at $\mathrm{pH}$ 1.0, 3.0 and 6.8 were gotten from the spectral data utilizing Ardon's plot. The qualities for security steady were ascertained from the inclines and captures of the straight lines from these plots. It was seen from the Ardon's condition that the estimations of solidness consistent was given as ((intercept)/(slope)) of straight line so got. i. e. $\mathrm{k}=$ (capture)/(incline). The estimation of intercept and slope were ascertained by Least Squares Method utilizing the accompanying equation: $\mathrm{y}=\mathrm{mx}+\mathrm{C}$

The values of stability constants for the drug-drug system at $\mathrm{pH} 1.0,3.0$ and 6.8 presented in the table 1.

\subsection{Drug-drug interaction measured by IRAffinity-1S FTIR spectrophotometer}

I R of the Vildagliptin, Bisoprolol fumarate was determined by FTIR spectra. Physical of drugs and their mixture were characterized by FTIR spectral analysis. From the results, it was concluded that there was moderate interference between Vildagliptin and Bisoprolol fumarate. Because a slight change of spectra occurred in their 1:1 complex. All of the spectra showed in Figure 4.

3.6 In vivo drug interactions study of Vildagliptin with Bisoprolol fumarate was evaluated by Oral Glucose Tolerance Test

All the treatments significantly reduce blood glucose level in $30 \mathrm{~min}$ after administration. Most significant reduction $(\mathrm{P}<0.01)$ was observed for 
Vildagliptin and the complex (2:1) at $120 \mathrm{~min}$. Other complexes (1:1 and $1: 2)$ also reduced the blood glucose level, but these are less than the $(2: 1)$ complex. Bisoprolol fumarate reduced negligible blood glucose level after 180 min of the treatment. All results are presented in Table 2, Figure 5 and Figure 6.

\subsection{In silico studies}

\subsubsection{Structural studies}

The structure of the complex of Vildagliptin with Bisoprolol fumarate found from the Accelrys Discovery Studio Visualizer was presented in Figure 7.

\subsubsection{NMR Prediction}

NMR Prediction from Chem office 2002 presented in Figure 8. In this figure, it is clear that the interaction between happened, because found structure of complex showed different NMR report then its mother compounds.

\subsubsection{DDI-CPI analysis}

The output results from DDI-CPI analysis showed in Table 3. In this analysis, Vildagliptin showed 1.000 interaction confidence towards Bisoprolol fumarate.

\section{Discussion}

Initial evidence for complexation of Vildagliptin with Bisoprolol fumarate originated from contrasts between the spectra of the medications and those of their mixtures in buffer solutions. Every compound has its interesting sub-atomic structure or electronic design which is in charge of assimilation of light. Clearly every compound has its one of a kind subatomic structure or electronic setup which is in charge of retention of light as bright or visible form (Saha et al.,2015, Ahsan, M. et al 2012). The molecular species of Vildagliptin and Bisoprolol fumarate when independently mixed demonstrated a few changes in retention attributes incorporating a few moves in the absorption maxima of buffer mixes and in addition their 1:1 mixture in buffer solution of $\mathrm{pH} 1.0,2.0$ and 6.8 at an altered focus (1 X 10-3) M. The modification in spectral pattern might be viewed as a marker for connection between these medications.

In Job's plot, at $\mathrm{pH} 1.0,2.0$ and 6.5, various concentrations comprising $1 \times 10^{-3} \mathrm{M}$ to $9 \times 10^{-3} \mathrm{M}$ of Vildagliptin were interacted with 
Bisoprolol fumarate. The breakdown in the curve at different concentrations of Vildagliptin indicates the presence of drug interaction.

The Ardon's plots have been utilized to assess the stability constants and it has been watched that when estimations of $1 /(\mathrm{D}-\mathrm{C} \varepsilon \mathrm{A})$ are plotted against 1/Drug (Figure 3), these lines are acquired complying with the Ardon's equation. The estimations of stability constants at various $\mathrm{pH}$ are appeared in table 3. Stability constants data demonstrated that Vildagliptin-Bisoprolol fumarate framework shaped generally more grounded buildings at all $\mathrm{pH}$ conditions.

The stability constants obtained from the Ardon's plot for the systems (Vildagliptin with Bisoprolol fumarate) were found to show mild drug interaction at $\mathrm{pH} 2.0$ and show stable complex at $\mathrm{pH} 1.0$ and show moderate stable complex at $\mathrm{pH} 6.8$ as the stability constant is greater than 1 , which is an indication of drug interaction.

The spectrum of 1:1 complex is slightly different from pure Vildagliptin and pure Bisoprolol fumarate. Spectrum of 1:1 complex is near to Bisoprolol fumarate. That means presence of Bisoprolol fumarate interrupt Vildagliptin spectrum. Because, when ever any type of interaction happened between two drugs, then different type of spectrum found. So IR report proved that, between Vildagliptin and Bisoprolol fumarate an interaction happen. But this interaction is moderate.

In this OGTT test at 1:1, 1:2 and 2:1 complex blood glucose level decrease compared to Vildagliptin. Because when drug-drug interaction happened, then drug cannot exhibit its main activity. That's why in this test complex showed different activity.

From the results of in silico methods used, they exhibited the presence of interaction by showing different parameters. In structural study of the drugs, they formed a complex, which conclude that they have attraction towards them. It also proved by other two methods also. In NMR prediction spectra, deviant spectra showed by the complex compound and DDI-CPI analysis also predicted that Vildagliptin had 1.000 interaction confidence towards Bisoprolol fumarate.

\section{Conclusion}

By all the trial information appeared above we can conclude that the interaction of Vildagliptin with Bisoprolol fumarate show diminish in free drug concentration of both drug which ultimately results in decrease affinity towards receptors and it is clear that one or both drug may show diminished pharmacological activity. The complex of Vildagliptin and Bisoprolol fumarate shows different spectra and in vivo process it was also causes difference in decrease of blood glucose level. All in vitro and in vivo 


\section{IIUC STUDIES, 14 (Issue 1, Special)}

report showed that Vildagliptin and Bisoprolol fumarate produced complex between them which can lower the concentration of free drug in plasma and may causes some undesirable effect. In in silico methods, results showed that the drugs interact with each other. It can in this way be presumed that a cautious thought is required during concurrent administration of Vildagliptin with Bisoprolol fumarate.

\section{Competing interests}

The authors declare that they have no competing interests.

\section{References}

Becker, M. L., Kallewaard, M., P. Caspers, W., Visser, L. E., Leufkens, H. G., et al. B. H. (2007). Hospitalisations and emergency department visits due to drug-drug interactions: A literature review. Pharmacoepidemiol Drug Saf, 16(6), 641-651.

Chou, T. C. (2006). Theoretical basis, experimental design, and computerized simulation of synergism and antagonism in drug combination studies. Pharmacol Rev, 58(3), 621-681.

Huang, S. M., \& Lesko, L. J. (2004). Drug-drug, drug-dietary supplement, and drug-citrus fruit and other food interactions: What have we learned?. J Clin Pharmacol, 44(6), 559-569.

van Roon EN, Flikweert S, le Comte M, Langendijk PN, Kwee-Zuiderwijk WJ et al .(2005). Clinical relevance of drug-drug interactions : A structured assessment procedure. Drug Saf, 28(12), 1131-1139.

Bergk ,V., Haefeli, W. E., Gasse, C., Brenner, H., Martin- Facklam, M., (2005). Information deficits in the summary of product characteristics preclude an optimal management of drug interactions: A comparison with evidence from the literature. European Journal of Clinical Pharmacology, 61(5-6), 327-335.

Chowdhury, K. A. A., Kabir, M. S. H., Chowdhury, T. A., Hossain, M. M., Kamal, A. T. M. M. et al. (2015). In vitro drug-drug interaction study between Montelukast Sodium and Amlodipine Besylate at gastric and intestinal pH. International Journal of Pharmacy, 5(3), 1003-1009.

Job., P. Job's method of continuous variation. Ann Chim, (1928). 10(9), 113.

Mohiuddin, M., Azam, ZAnn Chim, (1928). P. Job's method of continuous variation, 10(9), 113.., Amran, M. S., \& Hossain, M. A. (2009). In vitro study on the interaction of caffeine with gliclazide and metformin in the aqueous media. J. Pharmacol. Toxicol, 4(5), 194-204. 
Ardon, M. (1957). Oxidation of ethanol by ceric perchlorate. Journal of the Chemical Society (Resumed), 340, 1811-1815.

Sayeed, M. A., \& Rana, S. (2013). In vitro and invivo drug-drug interaction study between ketotifen fumerate and chlorpheniramine maleate at gastric and intestinal pH. e-Journal of Science \& Technology (e-JST), 8(2).

Xia, F., Xu, X., Zhai, H., Meng, Y., Zhang, H., Du, S., ... et al (2013). Castrationinduced testosterone deficiency increases fasting glucose associated with hepatic and extra-hepatic insulin resistance in adult male rats. Reproductive Biology and Endocrinology, 11(1), 1-11.

Luo, H., Chen, J., Shi, L., Mikailov, M., Zhu, H., Wang, K., ... et al. (2011). DRAR-CPI: A server for identifying drug repositioning potential and adverse drug reactions via the chemical-protein interactome. Nucleic acids research, W492-W498.

Saha, S., Begum, R., Rahman, A., Sultan, M. Z., Amran, M. S., \& Hossain, M. A. (2015). Evaluation of in vitro interaction of metformin with ibuprofen in aqueous medium. Bangladesh Pharmaceutical Journal, 16(2), 189-194.

Ahsan, M. R., Sultan, M. Z., Aamjad, F. M., Sultana, S., Baki, M. A., Hossain, M. A., ... et al. (2012). The study of in vitro interaction of ciprofloxacin with paracetamol and zinc in aqueous medium. Journal of Scientific Research, 4(3), 701-708.

Tables

Table 1: Stability constant of Vildagliptin with Bisoprolol fumarate at different $\mathrm{pH}$.

\begin{tabular}{lll}
\hline System & $\mathrm{pH}$ & Stability Constants \\
\hline Interaction of Vildagliptin & 1.0 & $1.8750^{* *}$ \\
with Bisoprolol fumarate & 3.0 & $0.7778^{*}$ \\
& 6.5 & $1.2000^{* *}$ \\
\hline
\end{tabular}

Values are the mean of triplicate experiments and represented as mean. Values in the same column with different superscripts $\left(^{*}\right)$ are significantly different $* \mathrm{P}<0.05$ and $* * \mathrm{P}<0.01$. Student's t test was performed to analyze this data set. 


\section{IIUC STUDIES, 14 (Issue 1, Special)}

Table 2: Effect of Vildagliptin with Bisoprolol fumarate and their mixtures of 1:1, 1:2 and $2: 1$, on glucose induced hyperglycemia $(\mathrm{mmol} / \mathrm{L})$ in normal mice.

\begin{tabular}{llllll}
\hline Group & 0 hour & 1st hour & 2nd hour & 3rd hour & $\begin{array}{c}\% \\
\text { decrease }\end{array}$ \\
\hline Control & $9.4 \pm 0.063$ & $9.96 \pm 0.25$ & $10.3 \pm 0.27$ & $9.74 \pm 0.144$ & - \\
Vildagliptin & $10.3 \pm 0.307$ & $8.46 \pm 0.835^{*}$ & $6.74 \pm 0.612^{*}$ & $7.48 \pm 0.406^{* *}$ & 27.38 \\
$\begin{array}{l}\text { Bisoprolol } \\
\text { fumarate } \\
\text { Mixture (1:1) }\end{array}$ & $9.24 \pm 0.845$ & $9.92 \pm 0.789^{*}$ & $9.24 \pm 0.64$ & $9.04 \pm 0.658$ & 2.16 \\
Mixture (1:2) & $9.32 \pm 0.26$ & $9.58 \pm 0.351$ & $8.92 \pm 0.373^{*}$ & $8.83 \pm 0.534 *$ & 5.26 \\
Mixture (2:1) & $10.24 \pm 0.299$ & $9.38 \pm 0.649 *$ & $9.1 \pm 0.378 *$ & $8.48 \pm 0.471 * *$ & 17.19 \\
\hline
\end{tabular}

Values are presented in mean \pm SEM $(n=5)$. Values with different superscripts in same column are significantly different from control at each specific hour after the administration of Vildagliptin with Bisoprolol fumarate and their mixture of different ratio. For ${ }^{*} P>0.05$ and ${ }^{* *} P<0.01$. One-way ANOVA followed by Dunnett's multiple comparison was performed to analyze this comparison. "-" means no decrease.

Table 3: Results from DDI-CPI analysis for Vildagliptin with Bisoprolol fumarate.

\begin{tabular}{lll}
\hline Input drug & Existant drug & Interaction confidence \\
\hline Vildagliptin & Bisoprolol fumarate & 1.000 \\
\hline
\end{tabular}


Figures
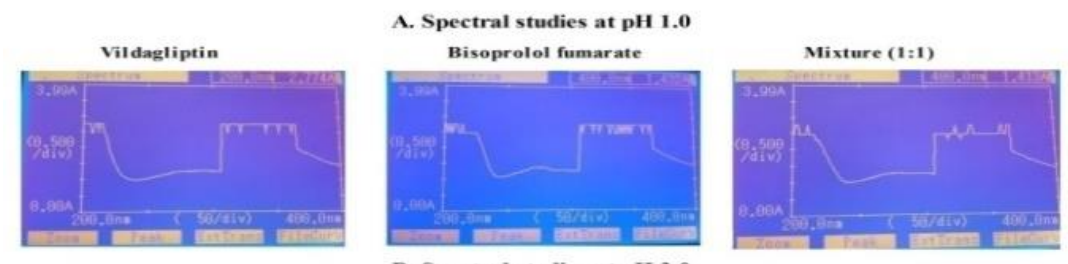

Vildagliptin

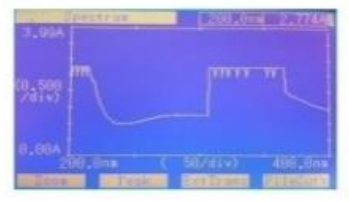

B. Spectral studies at pH 3.0 Biseprolel fumarate

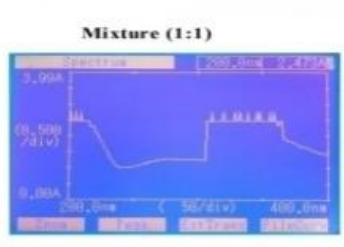

Vildagliptin

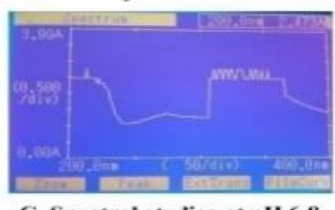

C. Spectral studies at pH 6.8 Bisoprolel fumarate

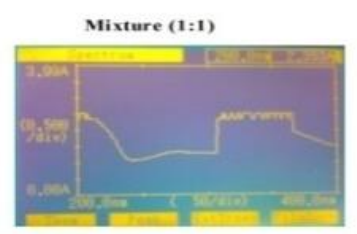

Figure 1: Spectral studies of Vildagliptin, Bisoprolol fumarate and their (1:1) mixture for A. $\mathrm{pH}$ 1.0, B. $\mathrm{pH} 3.0$, and C. $\mathrm{pH} 6.8$.

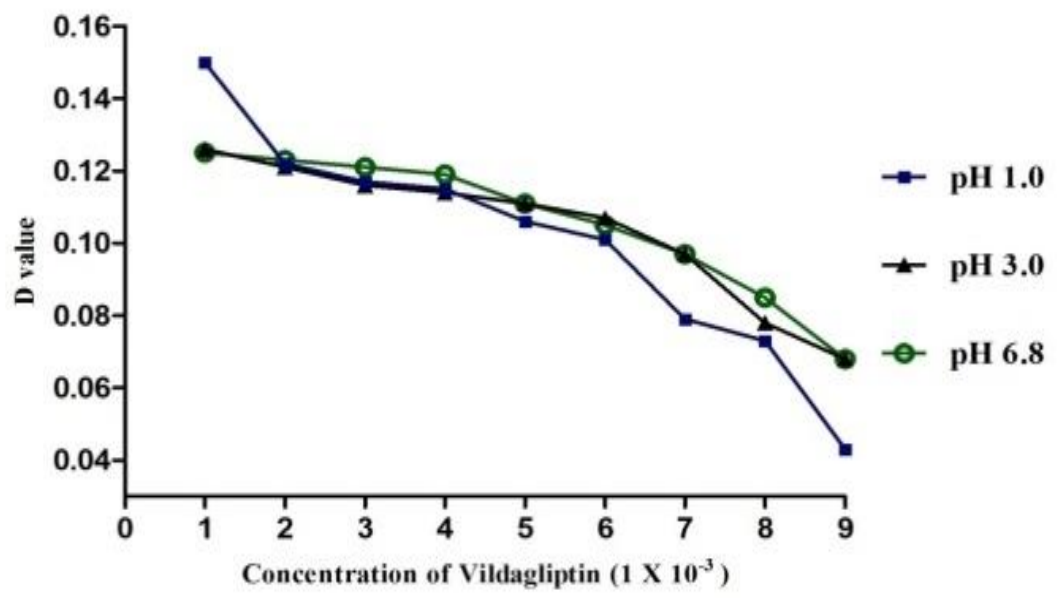

Figure 2: Job's plot for complexation of Vildagliptin with Bisoprolol fumarate at $244 \mathrm{~nm}$. 
A. Ardon's for pH 1.0

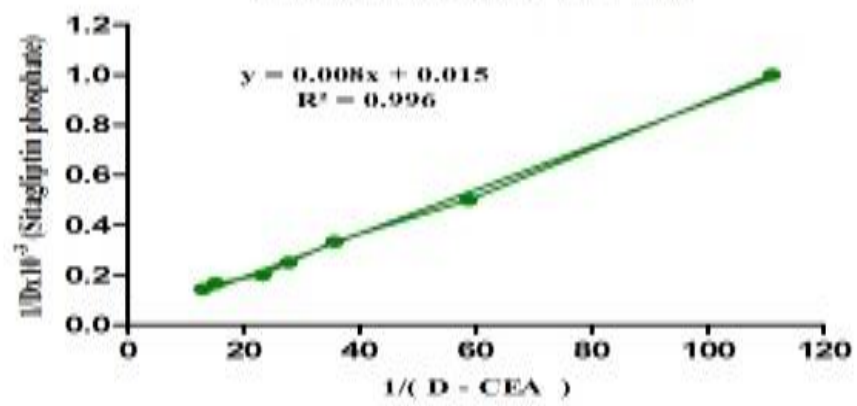

B. Ardon's for pH 3.0

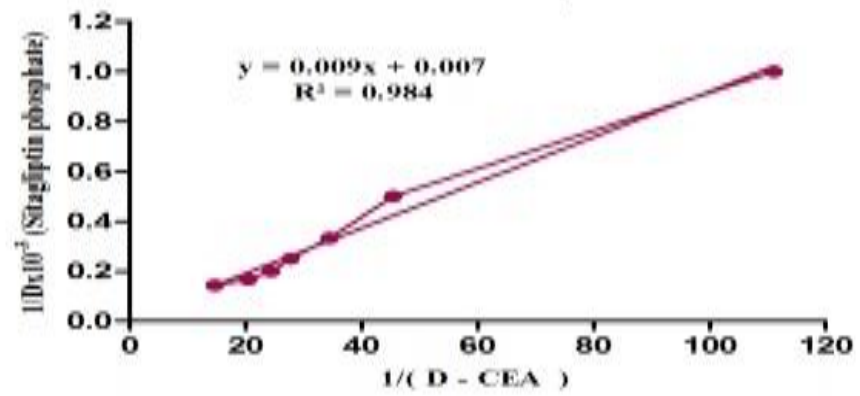

\section{Ardon's for pH 6.8}

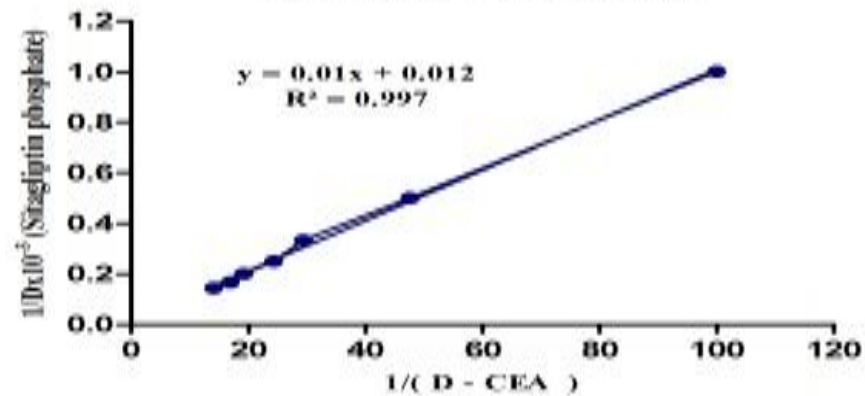

Figure 3: Ardon's plot for complexation of Vildagliptin with Bisoprolol fumarate at 244 nm for A. $\mathrm{pH} 1.0, \mathrm{~B} . \mathrm{pH} 3.0$ and C. $\mathrm{pH} 6.8$. 


\section{In vitro, in vivo and in silico 43}

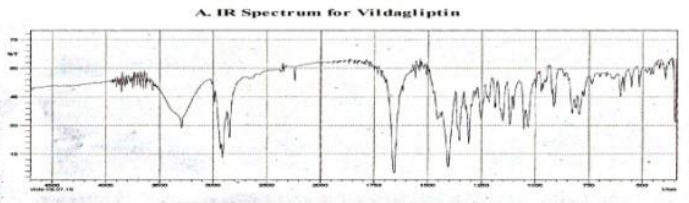

B. IR Spectrum for Bisoprolol rumarate
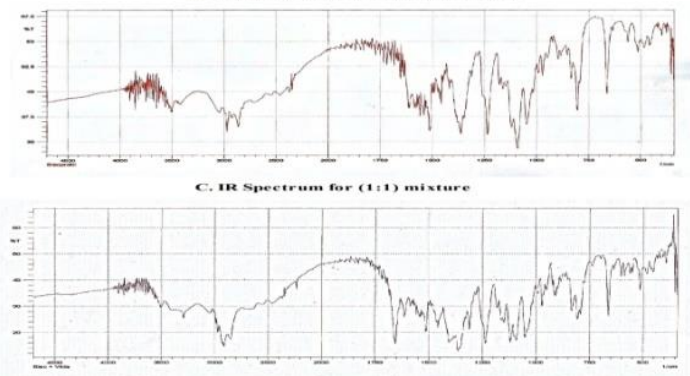

Figure 4: IR Spectrum for, A. Vildagliptin, B. Bisoprolol fumarate and C. their (1:1) mixture.

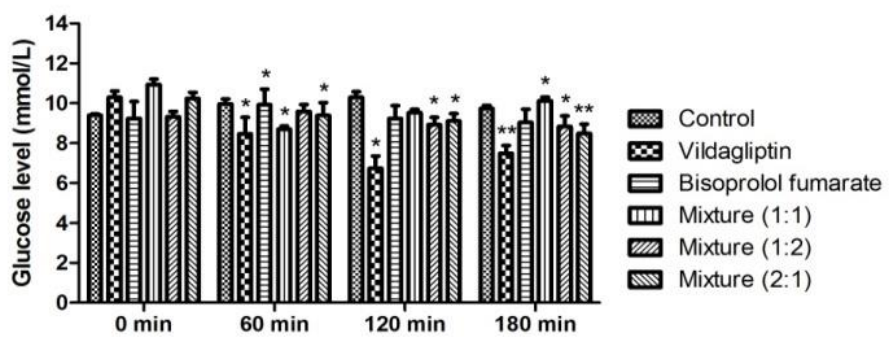

Figure 5: Effect of Vildagliptin with Bisoprolol fumarate and their mixtures of 1:1, 1:2 and 2:1 ratio, on glucose induced hyperglycemia $(\mathrm{mmol} / \mathrm{L})$ in normal mice. Values are presented in mean \pm SEM $(\mathrm{n}=5)$.

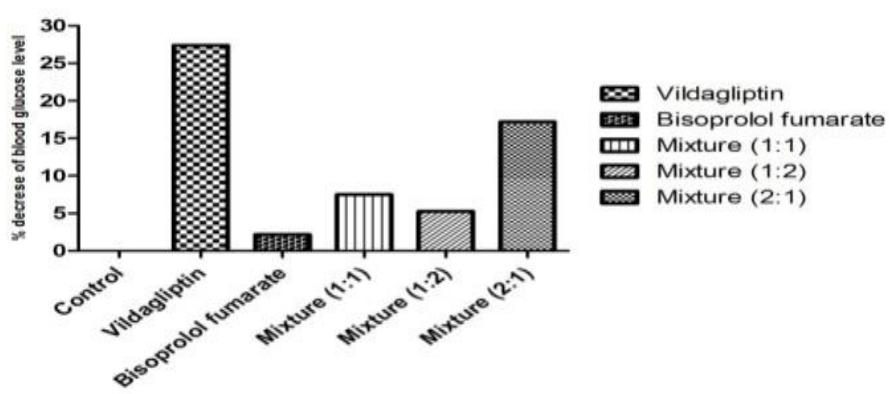

Figure 6: Percentage decrease of blood glucose level after treatment with Vildagliptin with Bisoprolol fumarate and their mixture of 1:1, 1:2 and 2:1 ratio, on glucose induced mice. 


\section{IIUC STUDIES, 14 (Issue 1, Special)}

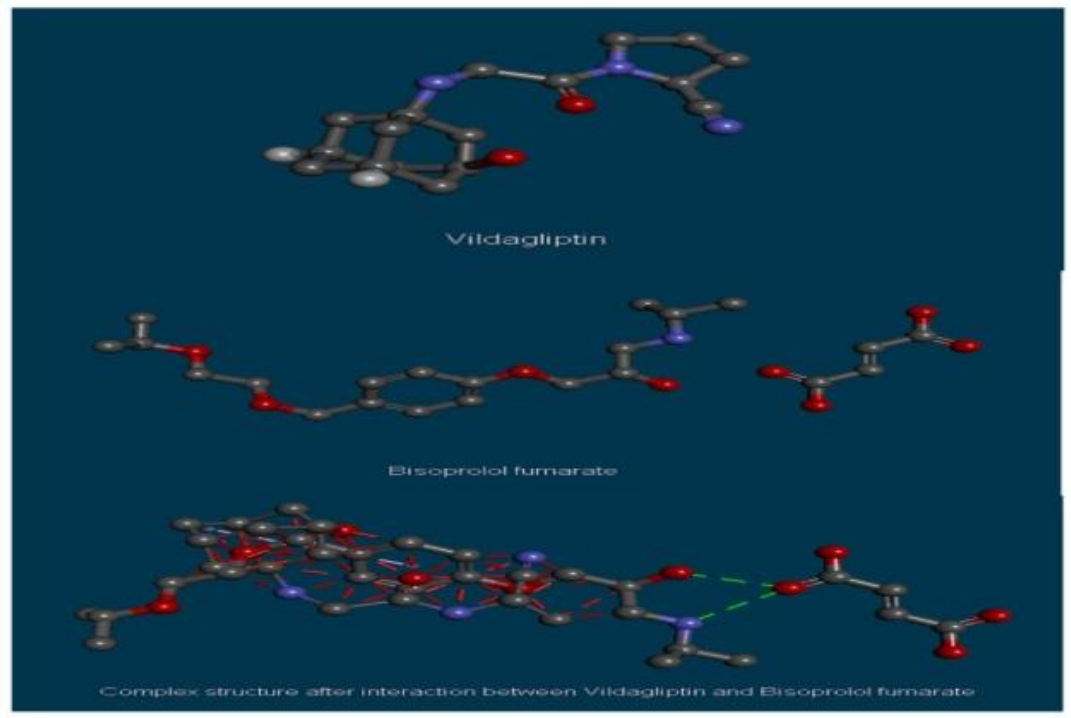

Figure 7: Structures of Vildagliptin, Bisoprolol fumarate and their interacted complex compound.

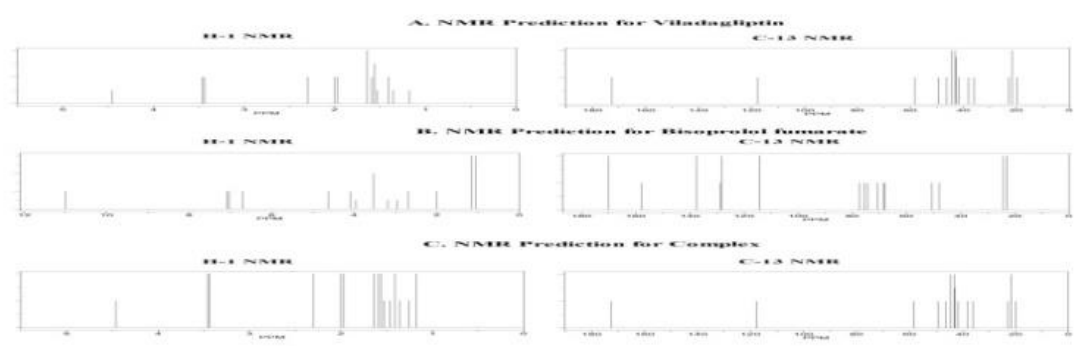

Figure 8: H-1 NMR and C-13 NMR prediction for, A. Vildagliptin, B. Bisoprolol fumarate and C. Complex of Vildagliptin and Bisoprolol fumarate.

\section{Corresponding author}

Kazi Ashfak Ahmed Chowdhury can be contacted at: ashfak4u_ctg@yahoo.com 